\title{
Foodborne Pathogens and Disease Special Issue on the National and International PulseNet Network
}

\author{
Stephen P. Oliver
}

$\mathbf{F}$ OODBORNE ILLNESS CONTINUES to be a huge issue and formidable challenge in the United States and throughout the world. The U.S. Center for Disease Control and Prevention (CDC) estimates that each year $\sim 1$ in 6 Americans or $\sim 48$ million people get sick, 128,000 are hospitalized, and 3000 die of foodborne diseases. In 2015, the World Health Organization (WHO) reported estimates of the global burden of foodborne diseases and indicated that 31 foodborne hazards were estimated to cause 600 million cases of foodborne disease and 420,000 deaths annually, worldwide. Given the considerable national/international demand for food safety and the formidable challenges of producing and maintaining a safe food supply, food safety continues to be of critical importance nationally and internationally.

The United States has an incredibly diverse and abundant food supply with a significant variety of foods imported from countries throughout the world. Foods that were once thought to be seasonal can now be obtained just about any time of the year. Ensuring the safety of both domestically produced and imported foods is extremely important and complex. The challenges to providing a safe and nutritious food supply are complex because all aspects of food production-from farm to fork-need to be considered. Not only must research and educational efforts be conducted to solve complex food safety problems but also results must be communicated to scientists; to the medical, veterinary, and agricultural communities; to public health and health care practitioners; to government regulatory agencies; to food producers and processors; and to consumers in a timely manner. This will ultimately help bridge the gap between science and instituting policy to reduce the burden of foodborne illness worldwide.

Preventing foodborne diseases requires comprehensive food safety systems and coordination among different government agencies involved in ensuring the safety of both domestically produced and imported foods. In the United States, federal and state agencies-including the Department of Health and Human Services' agencies, CDC and the Food and Drug Administration (FDA), and the Department of Agriculture's Food Safety and Inspection Service (USDA FSIS) - collaborate to ensure safety measures are met to protect the American people. The Food and Agriculture Organization of the United Nations (FAO) and WHO International Food Safety Authorities Network facilitate communication between $600+$ members from 188 of the 194 FAO and WHO Member States around the world. Effective com- munication networks within and between countries are invaluable to facilitate efficient food recalls or outbreak investigations and prevent national and international food safety emergencies.

A Special PulseNet Issue of Foodborne Pathogens and Disease was first published in 2006. PulseNet USA is the molecular surveillance network for foodborne disease in the United States. The network consists of state and local public health laboratories, as well as food regulatory agencies that follow PulseNet's standardized protocols to perform pulsedfield gel electrophoresis (PFGE). Since its inception in 1996, PulseNet has been instrumental in detection, investigation, and control of numerous outbreaks caused by Shiga toxinproducing Escherichia coli O157:H7, Salmonella enterica, Listeria monocytogenes, Shigella spp., Campylobacter, and others. The PulseNet network was replicated in different ways in Canada, Europe, the Asia Pacific region, Latin America, the Middle East, and Africa. These independent networks allowed public health officials to share molecular epidemiologic information in real time and better enabled rapid recognition and investigation of multinational foodborne disease outbreaks. Routine communication between the various international PulseNet networks provided early warning on foodborne disease outbreaks to participating public health institutions and countries.

Scientific advances have resulted in more accurate identification of the many foodborne pathogens that can be found in foods. For the past two decades, PFGE was the gold standard subtyping method for foodborne pathogens tracked by PulseNet. Although PFGE worked well for the intended purpose, the procedure has limitations for molecular characterization and subtyping of bacterial pathogens. A new gold standard is now being implemented with the introduction of cost-effective whole genome sequencing (WGS) for analysis of all the foodborne pathogens tracked by PulseNet. This is a major undertaking that affects every functional aspect of PulseNet, including laboratory workflows, data storage, analysis management and data interpretation, and language used to communicate information (sequence profile nomenclature system). This represents a major shift in workflows currently practiced in most public health laboratories, but one that brings opportunities for streamlining surveillance activities for the network as a whole.

This issue of Foodborne Pathogens and Disease focuses on advances in the PulseNet and related National and

Department of Animal Science, University of Tennessee, Knoxville. 
International programs since 2006 and should be of significant interest to the medical, public health, and research communities. How food regulatory agencies such as the FDA and USDA FSIS and their public health partners in the states and local jurisdictions, together with the CDC use WGS to support their mission of protecting Americans from illness caused by foodborne bacterial pathogens is described. In addition, it includes information on PulseNet programs in other countries and how these programs have been used to identify and characterize foodborne pathogen outbreaks.

In closing, this Special Issue represents significant scientific advances that have resulted in more accurate identification of the many foodborne pathogens that can be found in foods. This will ultimately have a major impact on food safety and reducing the global burden of foodborne diseases. I want to express my sincere appreciation to Dr. Peter Gerner-Smidt and his colleagues for their efforts in making this Foodborne Pathogens and Disease PulseNet Special Issue become a reality. I also thank all of the authors and coauthors who contributed review/overview and original research articles for the Foodborne Pathogens and Disease PulseNet Special Issue and to those who reviewed these articles.

Stephen P. Oliver, PhD Editor-in-Chief

Foodborne Pathogens and Disease Department of Animal Science University of Tennessee Knoxville, TN 37996

E-mail: soliver@utk.edu 